\title{
SARS-COV-2 Colonizes Coronary Thrombus and Impairs Heart Microcirculation Bed in Asymptomatic Positive Subjects with Acute Myocardial Infarction
}

raffaele marfella ( $\sim$ raffaele.marfella@unicampania.it)

Università degli Studi della Campania Luigi Vanvitelli https://orcid.org/0000-0003-3960-9270

\section{Pasquale Paolisso}

University of Naples Federico II: Universita degli Studi di Napoli Federico II

\section{Celestino Sardu}

University of Campania Luigi Vanvitelli: Universita degli Studi della Campania Luigi Vanvitelli

\section{Luciana Palomba}

University of Campania Luigi Vanvitelli: Universita degli Studi della Campania Luigi Vanvitelli

\section{Nunzia D'Onofrio}

University of Campania Luigi Vanvitelli: Universita degli Studi della Campania Luigi Vanvitelli

\section{Arturo Cesaro}

University of Campania Luigi Vanvitelli: Universita degli Studi della Campania Luigi Vanvitelli

\section{Michelangela Barbieri}

University of Campania Luigi Vanvitelli: Universita degli Studi della Campania Luigi Vanvitelli

\section{Maria Rosaria Rizzo}

University of Campania Luigi Vanvitelli: Universita degli Studi della Campania Luigi Vanvitelli

\section{Ferdinando Carlo Sasso}

University of Campania Luigi Vanvitelli: Universita degli Studi della Campania Luigi Vanvitelli

\section{Lucia Scisciola}

University of Campania Luigi Vanvitelli: Universita degli Studi della Campania Luigi Vanvitelli

\section{Fabrizio Turriziani}

University of Campania Luigi Vanvitelli: Universita degli Studi della Campania Luigi Vanvitelli

\section{Massimiliano Galdiero}

University of Campania Luigi Vanvitelli: Universita degli Studi della Campania Luigi Vanvitelli

\section{Danilo Pignataro}

University of Campania Luigi Vanvitelli: Universita degli Studi della Campania Luigi Vanvitelli

\section{Fabio Minicucci}

Cardarelli Hospital: Ospedale Cardarelli

\section{Maria Consiglia Trotta}

University of Campania Luigi Vanvitelli: Universita degli Studi della Campania Luigi Vanvitelli 


\section{Michele D'Amico}

University of Campania Luigi Vanvitelli: Universita degli Studi della Campania Luigi Vanvitelli

Ciro Mauro

Cardarelli Hospital: Ospedale Cardarelli

\section{Paolo Calabrò}

University of Campania "Luigi Vanvitelli", Italy

\section{Maria Luisa Balestrieri}

University of Campania Luigi Vanvitelli: Universita degli Studi della Campania Luigi Vanvitelli

\section{Emanuele Barbato}

University of Naples Federico II: Universita degli Studi di Napoli Federico II

\section{Marilena Galdiero}

University of Campania Luigi Vanvitelli: Universita degli Studi della Campania Luigi Vanvitelli

\section{Giuseppe Paolisso}

University of Campania Luigi Vanvitelli: Universita degli Studi della Campania Luigi Vanvitelli

\section{Research}

Keywords: intracoronary thrombus, SARS-COV-2, thrombus viral load, asymptomatic SARS-COV-2 patients, STEMI,

Posted Date: April 26th, 2021

DOI: https://doi.org/10.21203/rs.3.rs-443209/v1

License: (c) (i) This work is licensed under a Creative Commons Attribution 4.0 International License. Read Full License 


\section{Abstract}

Background. The viral load of asymptomatic SAR-COV-2 positive (ASAP) persons have been equal to that of symptomatic patients, suggesting a similar risk for endothelial dysfunction and increased coagulation in asymptomatic and symptomatic patients. To date, there are no reports of ST-elevation myocardial infarction (STEMI) outcomes in ASAP patients. We evaluated thrombus burden and thrombus viral load and their impact on microvascular bed perfusion in the infarct area (myocardial lush grade, MBG) in ASAP compared to SARS-COV-2 negative (SANE) STEMI patients.

Methods. This was an observational study of 46 ASAP, and 130 SANE patients admitted with confirmed STEMI treated with primary percutaneous coronary intervention and thrombus aspiration. The primary endpoints were thrombus dimension + thrombus viral load effects on MBG after PPCl. The secondary endpoints during hospitalization were major adverse cardiovascular events (MACEs). MACEs are defined as a composite of cardiovascular death, nonfatal acute AMI, and heart failure during hospitalization.

Results. Thrombus dimensions were significantly higher in ASAP patients as compared to SANE patients. Interestingly, 39 (84.9\%) of ASAP patients also had thrombus specimens positive for SARS-COV-2. In ASAP STEMI patients $(n=46)$, thrombus viral load was a significant determinant of thrombus dimension independently of risk factors $(p<0.005)$. MBG and left ventricular function were significantly lower in ASAP STEMI patients $(p<0.001)$. Multiple logistic regression analyses evidenced that thrombus SARSCoV-2 infection and dimension were significant predictors of poorer MBG in STEMI patients.

Conclusions. In ASAP patients presenting with STEMI, there is strong evidence towards higher thrombus viral load, dimension, and poorer MBG. These data support the need to reconsider ASAP status as a risk factor that may worsen STEMI outcomes.

\section{Introduction}

Since February 2020, there have been reports of infected persons with SARS-CoV-2 but did not develop symptoms of coronavirus disease 2019 (COVID-19), (1-3). Asymptomatic people appear to account for about $40-45 \%$ of SARS-CoV- 2 infections and may transmit the virus to others over a prolonged period, perhaps more than 14 days (1). Moreover, the asymptomatic infection may be associated with subclinical lung abnormalities, as detected by computed tomography (4). Interestingly, the viral load of such asymptomatic persons has been frequently found equal to that of symptomatic persons $(5,6)$, thus suggesting a similar risk for endothelial dysfunction and increased coagulation in asymptomatic and symptomatic patients. The role of SARS-COV-2 in leading endothelial dysfunction and increased coagulation is also well known in patients with acute myocardial infarction (AMI) (7). In fact, patients presenting with ST-Elevation Myocardial Infarction (STEMI) and concurrent COVID-19 infection evidenced a strong signal towards higher thrombus burden and poorer outcomes (7).

Moreover, these data are supported by the observations that the first AMI incidence was $\approx 5$ times higher during the acute phase of COVID-19 infection than the control (8). However, this study did not provide any 
evidence about the potential role of SARS-COV-2 infection on thrombus viral load and thrombus burden neither on clinical outcomes in asymptomatic SAR-COV-2 positive (ASAP) STEMI patients compared to SARS-COV-2 negative (SANE) STEMI patients. In this context, we hypothesized that SAR-COV-2 thrombus viral load, by increasing coagulative state and coronary thrombus dimension of culprit atherosclerotic lesion, may worsen clinical outcomes in ASAP patients presenting STEMI. Therefore, we evaluated thrombus viral load and thrombus burden and their impact on microvascular bed perfusion in the infarct area (Blush grade) in consecutive ASAP cases compared to SANE STEMI patients.

\section{Methods}

\section{Patient characteristics, angiographic and echocardiographic procedures evaluations}

This was a multicenter observational cohort study aimed to investigate the relationship between thrombus viral load, thrombus dimension, and in-hospital outcomes in ASAP STEMI patients. We examined patients with first STEMI treated with the primary percutaneous coronary intervention (PPCl) and thrombus aspiration (TA) without coronavirus disease 2019 (COVID-19) between February 2020 and November 2020. Patients with clinical evidence of COVID-19 were excluded from the study. All patients met the guideline definition of STEMI (9). Routine analyses were obtained on admission before coronary angiography and before the initiation of full medical therapy. All patients with STEMI had baseline serological samples before cardiac catheterization for full blood count, renal and liver function tests, Creactive protein, D-dimer, fibrinogen, lactate dehydrogenase ( $(\mathrm{DH})$, and high sensitivity (hs)-Troponin T.We considered eligible for the study all patients with: correspondence between ECG findings and suspected culprit artery; a minimum visual estimate of $50 \%$ stenosis in the culprit artery, and feasibility of performing TA, as judged by the treating physician; the age of 18 years or greater; presentation to the cardiac catheterization laboratory for PPCl in the setting of first STEMI. Patients with left ventricular ejection fraction less than $25 \%$, with previous myocardial infarction or previous $\mathrm{PPCl}$ and/or coronary bypass grafting, or who had received fibrinolytic therapy were instead excluded from the study.

Coronary angiography. Coronary angiography was performed either via the radial or femoral artery. The culprit lesion was identified and crossed with an angioplasty guidewire. During primary $\mathrm{PCl}$, unfractionated heparin was administered in a loading dose of 70-100 U/ kg with the activated clotting time (ACT) maintained $>250 \mathrm{sec}$. ACTs were recorded at 10-15 minute intervals after the initial dose of heparin. Glycoprotein (GP) Ilb/Illa inhibitors were used at the operator's discretion.

Thrombus burden. The thrombus content was classified by a modified TIMI Thrombus Grade Classification (10). This classification scores the thrombus in five grades: Grade 0 (G0): No angiographic characteristics of thrombus are present; Grade 1 (G1): Possible thrombus is present (reduced contrast density, haziness, or irregular lesion contour); Grade 2 (G2): There is definite thrombus, with greatest dimensions $\leq$ half the vessel diameter; Grade 3 (G3): Definite thrombus, with greatest linear dimension > half the vessel diameter but < 2 vessel diameters; Grade 4 (G4): Definite thrombus, with the largest 
dimension $\geq 2$ but $<4$ vessel diameters; Grade 5 (G5): Definite thrombus, with the largest dimension $\geq 4$ vessel diameters (2). To date, two experienced interventional cardiologists independently evaluated all angiographic parameters. Two independent pathologists, blinded to study protocol, evaluated the thrombus dimension. After TA, thrombus surface area was defined as the product of its length, height, and thickness. Therefore, the thrombus dimension was expressed as surface area in $\mathrm{mm}^{2}$.

Thrombus aspiration. 2017 Guidelines of the European Society of Cardiology for the management of acute myocardial infarction in patients presentingwithST-segment elevation (11) and 2018 Guidelines on myocardial revascularization (12) do not recommend routine use of thrombus aspiration(class III, level A). Nevertheless, the same guidelines state that thrombus aspiration may be considered in large residual thrombus burden cases. According to these recommendations, with the support of the flowchart proposed by Junhua Ge et al. (13), manual TA was performed based on angiographic selection criteria (e.g., the presence of a visible thrombus on angiography, large vessel easy to pass with the catheter, localization of the thrombus at the proximal or middle segments of the target vessel, TIMI Thrombus Grade Classification Grade G3-G5), followed by conventional PCI to the culprit's vessel. The TA started before crossing the lesion, with a minimum of two syringes $(40 \mathrm{~mL})$ of aspirate recommended. Investigators were appropriately trained to ensure that the guide catheter was engaged with the coronary ostia when removing the thrombectomy catheter. Finally, the guide catheter was aspirated after thrombectomy to avoid embolization of either air or thrombus from the guide catheter.

Myocardial blush grade. The Myocardial Blush Grade (MBG) was defined according to the Zwolle criteria (14). Angiographic assessment of myocardial reperfusion in patients treated with primary angioplasty for acute myocardial infarction: myocardial blush grade. However, we defined the blush grade into grades from 0 to 3. Thus, grade 0 indicated no myocardial blush or contrast density; or persistent "staining," suggesting leakage of the contrast medium into the extravascular space. The grade 1 blush was defined as minimal myocardial blush or contrast density). Grade 2 was indicative of moderate blush or obtained on the contralateral/ipsilateral non-IRA, and grade 3 was indicative of normal blush. The angiography studies were evaluated at 2 independent centers by experienced interventional cardiologists in both cases. The observers were blinded to the remaining clinical information. Laboratory 1 (Lab 1$)$ is the institution that carried out the study, the catheterization laboratory of a teaching hospital, and laboratory 2 (Lab 2), an independent catheterization core laboratory with extensive experience measuring the myocardial blush index. Grades 2 and 3 were considered normal perfusion. The interobserver variability was $10 \%$ for detecting grades 2 and 3 blush and $20 \%$ for grade 3 blush in Lab 1, 13\% and 15\%, respectively, in Lab 2.

Echocardiographic evaluation. In the current study, two experienced physicians in echocardiography performed a trans-thoracic two-dimensional echocardiogram with M-mode, conventional Doppler imaging (TDI) measurements in each patient admitted for acute ST-elevation myocardial infarction (STEMI). For the exams, we used a Philips iE33 echocardiography (Eindhoven, The Netherlands). Then, physicians acquired the images of echocardiography in the parasternal long and short-axis views. Thus, we calculated left ventricle end-diastolic diameter (LVEDD), end-diastolic volume (LVEDV), end-systolic 
diameter (LVESD), end-systolic volume (LVESV), and we determined left ventricle ejection fraction (LVEF) with the Simpson biplane method (ref). However, the physicians systematically performed averaged measurements in five consecutive samples to have final calculation measures. The physicians involved in the echocardiographic evaluation performed and analyzed each exam independently and blinded to the study protocol. Finally, two observers blinded to measures performed previously reviewed all measurements. The observers were blinded to study protocol (15).

The study follows the principles outlined in 1976, the Declaration of Helsinki, and its later amendments for the use of human tissue or subjects. The Institutional Review Board of University of Campania "Luigi Vanvitelli," Naples, Italy approved the protocol (Ethical Committee number 268 for study on SARS-COV-2, and number 151 for study TA).

\section{Nasal/pharyngeal swab and thrombus SARS-COV-2 analysis}

Post-catheterization, all patients underwent routine nasal/pharyngeal swab and thrombus analysis for the SARS-CoV-2 virus using real time-polymerase chain reaction (RT-PCR). The sample was dissolved by homogenization and then extracted by ripospinvrd kit(GeneAll). The RT-PCR was performed on CFX-96 Real-time PCR system (Bio-Rad) by Allplex 2019-nCoV assay, based on the analysis of four fluorophores: FAM for the revelation of E gene, Cal Red 610 for RdRP gene, Quasar 670 for $N$ gene, and HEX to analyze the internal control (IC). The result was evaluated through Seegene Viewer. Respiratory and thrombus specimens were collected by the local CDC and then shipped to designated authoritative laboratories to detect SARS-CoV-2 presence and load.

The viral load has been detected with cycle threshold $(\mathrm{CT})$ values $(5,16)$. CT values are the number of cycles needed to detect each genetic marker identified by real-time reverse transcription-polymerase chain reaction testing. A lower CT value indicates a higher amount of viral RNA. Paired values for each resident are depicted using a different shape. Positivity was defined as a cycle threshold (CT) value $\leq$ 38.0. Based on the admission SARS-CoV-2 RNA nasopharyngeal swab, patients were clustered in two groups: ASAPSTEMI patients (positive nasopharyngeal swab) and SANE STEMI patients (negative nasopharyngeal swab). Patients with laboratory-confirmed SARS-CoV-2 infection were defined as asymptomatic, who has no symptoms at the time of first clinical assessment and had no symptoms at the end of follow-up. The end of follow-up was defined as one or more negative respiratory specimen RTPCR test results.

\section{In-hospital outcomes}

The primary endpoints were thrombus dimension + thrombus viral load effects on microvascular bed perfusion in the infarct area (Blush grade) after PPCl. The secondary endpoints during hospitalization were major adverse cardiovascular events (MACEs). MACEs are defined as a composite of cardiovascular death, nonfatal acute AMI, and heart failure during hospitalization.

\section{Statistical analysis}


Descriptive statistical analyses were performed using SPSS Statistics version 23.0 (IBM). A 2-sided pvalue $<0.05$ defined statistical significance. Variables are expressed accounts (percentages), mean \pm standard deviation (SD), and median [lower quartile-upper quartile] as appropriate. Chi-squared analysis or Fisher's-exact test was used to compare categorical data between groups. The independent samples Student t-test or ANOVA test was used to compare normally distributed continuous data between groups, and the Mann-Whitney U-test was used to compare the distribution of continuous skewed data between groups.

Correlation performed using Pearson's correlation analysis and Spearman's correlation analysis in the case of skewed variables. Event rates were derived as Kaplan-Meier estimates and compared by log-rank test. Multivariate linear regression analyses were also used to test the independent association of SARSCoV-2 infection and thrombus viral load with thrombus dimension independently of age, sex, metabolic risk factors, hypertension, and smoking. Multiple logistic regression analysis was used to test the association of SARS-CoV-2 infection and thrombus dimension with MACE independently of age, sex, metabolic risk factors, hypertension, and smoking. Multiple logistic regression analysis was used to test the association of SARS-CoV-2 infection and thrombus dimension with MBG independently of age, sex, metabolic risk factors, hypertension, and smoking. The odds ratio and $95 \%$ confidence intervals $(\mathrm{Cl})$ were also calculated. A cluster analysis (composite score) allowed us to calculate a clustering of cardiometabolic risk factors and use it as a covariate. For this purpose, we created a compound score referred to as a clustering score. It was calculated as the sum of Z-scores of the main cardio-metabolic risk factors (BMI, plasma glucose, triglycerides, total, LDH, and HDL cholesterol levels). A z-score indicates the position of an individual value of a variable in the total distribution of the variable in the population and is calculated as follows: (individual value - mean value)/ standard deviation. The sample size, estimated according to a global effect size of $27 \%$ with type I error of 0.05 and a power of $90 \%$, was 140 patients.

\section{Results}

Patient characteristics. 777 patients with acute myocardial infarction were admitted at cardiologic study centers between February 2020 to November 2020. Of these, 474 (61.1\%)were hospitalized for STEMI and 303 for No ST-elevation myocardial infarction (NSTEMI). Consequently, 474 were considered eligible STEMI patients and underwent the angiographic study. After the coronary study, 8 patients were excluded due to CABG indication, 3 for the absence of coronary lesions, while287 were not treated with TA. Thus, the study population consisted of 176 consecutive patients with confirmed STEMI, submitted to PPCI and TA, admitted during 40 weeks. Of the 176 patients, 46 (26.1\%) were ASAP patients, while 130 (73.9\%) patients were SANE patients. In ASAP patients, the viral load was $26 \pm 8 \mathrm{CT}$. Interestingly, 39 (84.9\%) of asymptomatic patients with SARS-COV-2 positive respiratory specimens had also thrombus specimens positive for SARS-COV-2. SANE STEMI patients were more likely than ASAP STEMI patients to be diabetic (29.2 vs. 17.4, $\mathrm{P}=0.478$ ), hypertensive ( 55.4 vs. $39.1, \mathrm{P}=0.042)$, and have higher body mass index (BMI) and age (Table 1). ASAP patients had higher thrombus dimension (A), hs-Troponin (B), D-dimer (C), and Creactive protein (D) and levels than SANE patients (Fig. 1). Interestingly, we evaluated thrombus viral load in 12 patients asymptomatic at screening, but that developed COVID-19 during hospitalization (not 
included in the evaluation). The thrombus viral load was similar to the ASAP patients in these patients (Fig. 2). 
Table 1

Clinical and instrumental characteristic of study population

\begin{tabular}{|c|c|c|c|}
\hline & \multirow{2}{*}{$\begin{array}{l}\text { Asymptomatic SARS-COV-2 } \\
\text { Positive patients }(\mathrm{N}=46)\end{array}$} & SARS-COV-2 & \multirow[t]{2}{*}{$\mathbf{P}$} \\
\hline & & Negative patients $(\mathrm{N}=130)$ & \\
\hline Age, years & $56.13 \pm 6.21$ & $68.43 \pm 6.46$ & 0.006 \\
\hline Male, n (\%) & $31(67.4)$ & $86(66.2)$ & 0.515 \\
\hline $\mathrm{BMI}, \mathrm{kg} / \mathrm{m}^{2}$ & $27.09 \pm 1.81$ & $29.55 \pm 1.97$ & 0.003 \\
\hline Diabetes,n (\%) & $8(17.4)$ & $38(29.2)$ & 0.478 \\
\hline Dyslipidemia, n (\%) & $7(15.2)$ & $30(23.7)$ & 0.181 \\
\hline Hypertension, n (\%) & $18(39.1)$ & $72(55.4)$ & 0.042 \\
\hline Smoking, n (\%) & $3(6.5)$ & $39(29.2)$ & 0.001 \\
\hline Acetylsalicylic acid, n (\%) & $21(45.6)$ & $60(46.1)$ & 0.546 \\
\hline Ace-inhibitors, n (\%) & $15(32.6)$ & $45(34.6)$ & 0.477 \\
\hline AT II receptor blocker & $6(13.1)$ & $25(19.2)$ & 0.239 \\
\hline Insulin, n (\%) & $2(4.3)$ & $5(3.8)$ & 0.587 \\
\hline Oral anti-diabetic drugs, n (\%) & $6(13.1)$ & $30(23.1)$ & 0.106 \\
\hline Statin, n (\%) & $7(15.2)$ & $27(20.8)$ & 0.278 \\
\hline Glycemia, mg/dl & $129.65 \pm 21.02$ & $129.22 \pm 27.64$ & 0.924 \\
\hline Cholesterol, mg/dl & $181.2 \pm 21.4$ & $197.7 \pm 21.1$ & 0.001 \\
\hline LDL-cholesterol, mg/dl & $111.57 \pm 21.65$ & $123.83 \pm 20.43$ & 0.010 \\
\hline Triglycerides, mg/dl & $163.91 \pm 23.63$ & $181.79 \pm 18.06$ & 0.001 \\
\hline LDH, unit/I & $367.83 \pm 37.41$ & $375.46 \pm 33.32$ & 0.126 \\
\hline Creatinine, $\mathrm{mg} / \mathrm{dl}$ & $0.92 \pm 0.22$ & $1.04 \pm 0.14$ & 0.001 \\
\hline Fibrinogen, g/l & $3.68 \pm 0.37$ & $3.75 \pm 0.33$ & 0.198 \\
\hline White cells count, $10^{9} / \mathrm{l}$ & $15.76 \pm 1.27$ & $12.49 \pm 4.82$ & 0.002 \\
\hline Lymphocyte, $10^{9} / /$ & $1.76 \pm 0.20$ & $1.80 \pm 0.14$ & 0.130 \\
\hline Virus load CT, n & $26.02 \pm 2.22$ & / & / \\
\hline $\begin{array}{l}\text { Data are means } \pm S D \text { or } n(\%) \text {. } \\
\text { descending artery; } L M S=\text { left } n \\
\text { circumflex artery; } R C A=\text { right } c \\
\text { infarction. }\end{array}$ & $\begin{array}{l}\mathrm{MI}=\text { body mass index; } \mathrm{CT}=\mathrm{c} \\
\text { in coronary artery; MACE: ma } \\
\text { onary artery; } \mathrm{ACT}=\text { activated }\end{array}$ & $\begin{array}{l}\text { e threshold; } \mathrm{LAD}=\text { left anterior } \\
\text { r adverse cardiac events; } \mathrm{Cx}= \\
\text { tting time; } \mathrm{AMI}=\text { acute myoca }\end{array}$ & dial \\
\hline
\end{tabular}




\begin{tabular}{|c|c|c|c|}
\hline & Asymptomatic SARS-COV-2 & SARS-COV-2 & P \\
\hline & Positive patients $(\mathrm{N}=46)$ & Negative patients $(\mathrm{N}=130)$ & \\
\hline Thrombus virus load CT, $\mathrm{n}$ & $25.46 \pm 8.68$ & I & / \\
\hline Ejection fraction, \% & $41.49 \pm 1.82$ & $47.25 \pm 3.55$ & 0.003 \\
\hline LAD, n (\%) & $31(67.4)$ & $74(56.9)$ & 0.091 \\
\hline LMS, n (\%) & $8(17.4)$ & $15(11.3)$ & 0.145 \\
\hline $\mathrm{Cx}, \mathrm{n}(\%)$ & $5(10.9)$ & $13(10.0)$ & 0.520 \\
\hline RCA, n (\%) & $12(26.1)$ & $39(30.0)$ & 0.349 \\
\hline GP Ilb/Illa inhibitor, n (\%) & $25(54.3)$ & $19(14.6)$ & 0.006 \\
\hline Multi-vessel PCl, n (\%) & $10(21.7)$ & $11(8.5)$ & 0.020 \\
\hline Thrombus dimension, $\mathrm{mm}^{2}$ & $4.21 \pm 1.12$ & $2.71 \pm 0.42$ & 0.001 \\
\hline Modified thrombus G 5, n (\%) & $41(89.1)$ & $30(23.1)$ & 0.001 \\
\hline Multivessel thrombus, n (\%) & $16(34.8)$ & $12(9.2)$ & 0.001 \\
\hline Post-PPCI TIMI 3, n (\%) & $43(93.5)$ & $124(95.4)$ & 0.432 \\
\hline Blush grade $2-3, \mathrm{n}(\%)$ & $12(26.1)$ & 127 (97.7) & 0.001 \\
\hline Total heparin dose, $\mathrm{U}$ & $12777.78 \pm 356$ & $10137.55 \pm 299$ & 0.047 \\
\hline Door-to-balloon time, min & $54(38-72)$ & $51(35.3-57.9)$ & 0.345 \\
\hline Average ACT & $268.7 \pm 67.2$ & $271.6 \pm 59.3$ & 0.167 \\
\hline MACE: & & & \\
\hline Death, n (\%) & $4(8.7)$ & $2(1.5)$ & 0.041 \\
\hline Non fatal AMI, n (\%) & $6(13.0)$ & $4(3.1)$ & 0.021 \\
\hline Heart failure, n(\%) & $4(8.7)$ & $3(2.3)$ & 0.077 \\
\hline $\begin{array}{l}\text { Data are means } \pm \text { SD or } n(\%) \text {. } \\
\text { descending artery; LMS = left } \\
\text { circumflex artery; RCA = right c } \\
\text { infarction. }\end{array}$ & $\begin{array}{l}\mathrm{Al}=\text { body mass index; } \mathrm{CT}=\mathrm{C} \\
\text { in coronary artery; MACE: } \mathrm{m} \\
\text { onary artery; } \mathrm{ACT}=\text { activated }\end{array}$ & $\begin{array}{l}\text { threshold; } L A D=\text { left anterior } \\
\text { adverse cardiac events; } C x= \\
\text { tting time; } A M I \text { = acute myoca }\end{array}$ & dial \\
\hline
\end{tabular}

Procedural characteristics. All patients underwent a primary PCI procedure and TA in both groups. Time from symptoms to reperfusion and ECG presentations were similar in both groups. Median door to balloon times was within $66 \pm 16$ minutes and similar for both groups. All patients received a loading dose of aspirin $300 \mathrm{mg}$ and either clopidogrel $600 \mathrm{mg}$ or ticagrelor $180 \mathrm{mg}$ before the procedure. All patients then received $100 \mathrm{mg}$ aspirin per day plus either $75 \mathrm{mg}$ clopidogrel per day or $90 \mathrm{mg}$ ticagrelor twice-daily maintenance therapy. Interestingly, there were no differences between the ASAP group and the 
SANE group in the \% stenosis of the coronary lesion after thrombectomy but before stenting. There was evidence of higher thrombogenicity in the ASAP patients with significantly higher rates of modified thrombus grade 5 (Table 1) and thrombus dimension (Fig. 1A). Myocardial blush grade and left ventricular ejection fraction were significantly lower in the ASAP patients than the SANE patients (Table 1).In keeping with this, there was significantly greater use of GP Ilb/IIlainhibitors $(p<0.0001)$, and to reach the similar ACTs total dose of heparin was higher in the ASAP group (Table 1). In all patients, thrombus dimension correlated with ejection fraction $(r=0.475, p<0.001)$. In asymptomatic patients, thrombus viral load correlated with thrombus dimension $(r=0.365, p<0.001$;Figure $3 A)$, hs-Troponin levels $(r=0.3414, p<0.001$;Figure 3B) and ejection fraction $(r=0.286, p<0.001$; Figure 3C). This latter correlation was also independent of age, smoking, and metabolic factor composite score $(r=0.201, p<0.03)$.

In-hospital outcomes. After PPCl, a blush grade post-PPCl 2-3 was present in only $26.1 \%$ of ASAP vs. $97.7 \%$ of SANE STEMI patients $(P<0.001)$. Kaplan-Meyer analysis in ASAP STEMI patients during hospitalization following PPCI showed a significantly higher in-hospital survival for MACE than in SANE STEMI patients $(p<0.001$; Fig. 2D). Moreover, among MACE outcomes, death and nonfatal AMI were higher in ASAP than in SANE patients (Table 1).In all study population (176), multivariate linear regression analysis, with thrombus dimension as the dependent variable, evidenced that SARS-CoV-2 infection and metabolic risk factors, as a composite score, were independent predictors of thrombus dimension (Table 2). The predictive role of SARS-CoV-2 infection and thrombus dimension on MBG outcome was tested in multiple logistic regression analyses (Table 3). Both SARS-CoV-2 infection (Model A) and thrombus dimension (Model B) were significant predictors of poorer blush grade outcomes in STEMI patients $(n=176)$. A model including both SARS-CoV-2 viral load and thrombus dimension revealed a main independent effect of SARS-CoV-2 infection and MBG (Model C) (Table 3).In ASAP STEMI patients $(n=46)$, thrombus viral load was a significant predictor of thrombus dimension independently of age, sex, smoking, hypertension, and metabolic risk factor composite score $(\beta=1.08 ; t=$ 5.40; $p=0.0001)$ ). Indeed, categorizing ASAP patients $(n=46)$ by gender, female had higher thrombus viral load $(15.53 \pm 4.5$ vs $30.25 \pm 5.51 \mathrm{CT} ; \mathrm{p}<0.001)$ and thrombus dimension $(4.62 \pm 0.44$ vs $4.00 \pm 1.28$ $\mathrm{mm} 2 ; \mathrm{p}<0.001)$ compared to male subjects. In contrast, no difference by gender in thrombus dimensions was found in all study population $(n=176)$. 
Table 2

Multivariate linear regression analysis with thrombus dimension as dependent variable $(\mathrm{n}=$ 176)

\begin{tabular}{|llllll|}
\hline Variables & $\boldsymbol{B}$ & Errore std. & Beta & $\boldsymbol{t}$ & $\boldsymbol{p}$ \\
\hline Age & .009 & .008 & .081 & & \\
\hline Sex & -.271 & .139 & -.136 & -1.943 & .054 \\
\hline SARS-CoV-2 infection & 1.439 & .170 & .674 & 8.451 & .000 \\
\hline Metabolic risk factor composite score & -.051 & .023 & -.144 & -2.234 & .027 \\
\hline Hypertension & .064 & .119 & .034 & .539 & .591 \\
\hline Smoking & -.091 & .142 & -.041 & -.642 & .522 \\
\hline
\end{tabular}

Table 3. Multiple logistic regression analyses with Blush grade as dependent variable $(n=176)$ 


\begin{tabular}{|c|c|c|c|c|}
\hline MODEL A & B & Sign. & $\operatorname{Exp}(B)$ & 95\% C.I.per EXP(B) \\
\hline Age & .060 & .066 & 1.062 & $.996-1.132$ \\
\hline Sex & .368 & .536 & 1.445 & $.450-4.639$ \\
\hline Metabolic risk factor Composit score & .161 & .109 & 1.175 & $.964-1.432$ \\
\hline Hypertension & .621 & .246 & 1.862 & $.651-5.319$ \\
\hline Smoking & .779 & .253 & 2.179 & $.574-8.277$ \\
\hline Thrombus dimension & -.873 & .001 & .418 & $.250-.699$ \\
\hline MODEL B & B & Sign. & $\operatorname{Exp}(B)$ & 95\% C.I.per EXP(B) \\
\hline Age & -.129 & .023 & .879 & $.786-.982$ \\
\hline Sex & 1.231 & .167 & 3.426 & $.599-19.607$ \\
\hline Metabolic risk factor Composit score & -.039 & .781 & .961 & $.729-1.269$ \\
\hline Hypertension & .011 & .988 & 1.011 & $.240-4.250$ \\
\hline Smoking & -.432 & .663 & .649 & $.093-4.525$ \\
\hline SARS-CoV-2 infection & -7.287 & .000 & .001 & $.000-.010$ \\
\hline MODEL C & B & Sign. & $\operatorname{Exp}(B)$ & 95\% C.I.per EXP(B) \\
\hline Age & -.134 & .022 & .875 & $.780-.981$ \\
\hline Sex & 1.293 & .149 & 3.643 & $.631-21.048$ \\
\hline Metabolic risk factor Composit score & -.025 & .862 & .975 & $.736-1.293$ \\
\hline Hypertension & .021 & .977 & 1.022 & $.240-4.350$ \\
\hline Smoking & -.499 & .610 & .607 & $.089-4.122$ \\
\hline Thrombus dimension & -.785 & .001 & .413 & $.247-.689$ \\
\hline SARS-CoV-2 infection & -3.234 & .518 & .039 & $.000-714$ \\
\hline
\end{tabular}

\section{Discussion}

This study represents the first comparative data to describe the coronary thrombus viral load and dimension in ASAP patients presenting with STEMI. This analysis demonstrates increased thrombus dimension in ASAP STEMIpatients compared with STEMI patients who are not infected. Interestingly, 39 (84.9\%) of asymptomatic patients with SARS-COV-2 positive respiratory specimens also had coronary thrombus specimens positive for SARS-COV-2. These observations fit a higher incidence of multiple thrombotic culprit lesions as well as lower MBG. Consistent with this, lower left ventricular systolic function and increased troponin levels were observed in ASAP STEMI patients despite similar median 
ischemia times.ASAP STEMI patients more often had blood abnormalities reflecting a systemic inflammatory response (elevated D-dimers and C-reactive protein levels) compared with SANE patients. Although SANE STEMI patients more often had diabetes, hypertension, and obesity as well as were older, higher rates of poor blush grade $(<2)$ were seen in ASAP patients, suggesting the extent of damage of the microvascular bed (12). In fact, despite more than $90 \%$ of ASAP STEMI patients presented restoration of epicardial blood flow after PPCI (TIMI grade flow 3 ), only $26 \%$ of ASAP patients had blush grade postPPCI 2-3. The MBG is associated with ST-segment elevation resolution, enzymatic infarct size, left ventricular function, and long-term mortality (17). Interestingly, there were no differences between the ASAP group and the SANE group in the \% stenosis of the coronary lesion after thrombectomy but before stenting. This observation suggests that in agreement with the difference in thrombus viral load, the lesions appear different about thrombus burden, but not in the degree of vessel stenosis, suggesting that ASAP and SANE lesions were only different regarding coagulation burden.Accordingly, we observed a reduced survival from in-hospital mortality, nonfatal AMI, and heart failure were seen in ASAP patients compared to SANE patients. Although these data were obtained in a relatively small number of ASAP STEMI patients, the reduced survival may be supported as an outcome by the blush grade evaluation that evidenced reduced perfusion in the microvascular bed of ASAP patients. Intriguingly, coronary thrombus viral load was found to correlate with thrombus dimension and troponin T levels, suggesting an important role of SARS-COV-2 thrombus colonization in poorer outcomes of ASAP STEMI patients. Here we describe coronary thrombus assessment features such as thrombus viral load and dimension, which raise the suspicion of the active role of SARS-COV-2 in the pro-thrombotic state of ASAP STEMI patients. Since STEMI was the first manifestation of the disease in this cohort and ASAP patients had lower cardiovascular risk factors than SANE patients, our data suggest that presentation of STEMI might itself be considered a thrombotic complication of SARS-COV-2 dissemination through endothelial cells and thrombus. Accordingly, thrombus viral load was a significant predictor of thrombus dimension independently of age, sex, smoking, hypertension, and the compound score of metabolic risk factors. Moreover, the predictive role of SARS-CoV-2 infection and thrombus dimension on blush grade outcome was tested in multiple logistic regression analyses suggesting that SARS-CoV-2 infection and thrombus dimension were significant predictors of adverse clinical outcome in STEMI patients. Taken together, these data raise the doubt that asymptomatic status in SARS-COV-2 positive patients might affect STEMI outcomes as it may have major implications in patient management. As background for these associations, pre-COVID data regarding the influenza virus suggest that patients with acute respiratory infections were at significantly elevated risk for developing atherosclerotic plaque rupture leading to myocardial infarction, the profound inflammatory response, and hemodynamic changes (18). These previously reported features are associated with higher coronary thrombus burden and poorer outcomes in COVID-19 cohorts with STEMI (19-21). COVID-19 infection is associated with a pro-thrombotic state (22). The occurrence of venous thrombus-embolic complications, both clinically apparent and subclinical, appears to be an important manifestation of the COVID-19 and related to disease severity and outcome (23). Increased thrombogenicity in acute ischemic stroke has also been described (24). Moreover, emerging data from large COVID-19 cohorts without STEMI (25) suggests that anticoagulation confers mortality benefit in this patient group. However, there have been no reports of increased coronary artery 
thrombus dimension, thrombus SARS-COV-2 colonization, and poorer blush grade outcomesinASAP patients presenting with STEMI.Mechanisms that trigger a presentation with STEMI and its associated higher arterial thrombus burden in ASAP patients are unknown. Relative to venous thromboembolism, arterial thrombus formation is more likely to be due to platelet activation and endothelial dysfunction. SARS-CoV-2 causes a systemic inflammatory response, leading to endothelial and hemostatic activation, including the activation of platelets and the coagulation cascade (18). In keeping with this, the data presented here show significantly higher rates of CPR, D-dimer, and Troponin I levels in the ASAP patients suggesting that this condition may also confer an increased risk of poorer blush grade outcome in ASAP patients. Mechanisms for this might include increased endothelial dysfunction or their effects on the immune system $(26,27)$. Whether these alterations may be responsible for poor outcomes in ASAP patients is a point to be confirmed with further studies. However, the similar viral load evidenced in asymptomatic and COVID-19 patients could raise the doubt that, also in asymptomatic patients, SARSCOV-2 can produce endothelial damage and increase coagulation (Fig. 4).

Study Limitations. It is a relatively small observational study and has all the limitations of this type of analysis, including bias and confounding potential. However, our objective was to evaluate thrombus viral load and thrombus burden as well their impact on microvascular bed perfusion in the infarct area (Blush grade, MBG) in asymptomatic SAR-COV-2 positive compared to SARS-COV-2 negative STEMI patients, and not to evaluate the effects of thrombus aspiration and/or treatment and/or a drug on clinical outcomes, objectives that require a randomized trial. Moreover, patient selection is in accordance with the routine clinical practice, as patients underwent thrombus aspiration based on 2017 AHA guidelines (28) and ESC 2017 (29) to manage the patient with STEMI. Accordingly, the percentage of patients undergoing thrombus aspiration in our study ( 176 of $474,37 \%$ ) agrees with previous and more numerous observational studies (30-32).

\section{Conclusion}

We evidenced thatASAPhad higher thrombus viral load and greater thrombus dimension than SANE patients; such data in ASAP patients were also associated with poorer blush grade outcomes compared toSANE patients presenting with STEMI. The strong signal towards significantly higher thrombus viral load and higher thrombus dimension is a novel finding that raises the question of more aggressive antithrombotic therapy in patients at high risk for cardiovascular diseases, as diabetic, hypertensive, and dyslipidemic ASAP patients. These observations may be helpful for considering the asymptomatic SARSCOV-2 positive condition as a clinical state that may influence atherosclerotic patients' cardiovascular outcomes. Further studies are needed to confirm this critical finding.

\section{Declarations}

Acknowledgments: Not applicable. 
Authors' contributions: Conception, design and interpretation of data: RM and GP; Acquisition of data: PP, CS, MRR, FCS, FT, FM, PC.

Analysis of data: MLB, NDO, MG; LP, MCT, MD, LS, MB, DP, MG, EB.

All authors read and approved the final manuscript

Funding: This study was funded by the National Research Program of Italy (PRIN2017).

Availability of data and materials: The datasets used and analyzed during the current study are available from the corresponding author on reasonable request.

Ethical Committee number: 268 for study on SARS-COV-2, and number 151 for study TA.

Consent for publication: not applicable

Competing interests: The authors declare that they have no competing interests.

\section{References}

1. Oran DP, Topol EJ. Prevalence of Asymptomatic SARS-CoV-2 Infection: A Narrative Review. Ann Intern Med. 2020;173:362-7. doi:10.7326/M20-3012.

2. Nishiura $\mathrm{H}$, Kobayashi T, Miyama T, et al. Estimation of the asymptomatic ratio of novel coronavirus infections (COVID-19). Int J Infect Dis. 2020;94:154-5. doi:10.1016/j.jiji.2020.03.020.

3. Bai Y, Yao L, Wei T, et al. Presumed asymptomatic carrier transmission of COVID-19. JAMA. 2020. doi:10.1001/jama.2020.2565.

4. Inui S, Fujikawa A, Jitsu M, et al. Chest CT findings in cases from the cruise ship "Diamond Princess" with coronavirus disease 2019 (COVID-19). RadiolCardiothorac Imaging. 2020;2. doi:10.1148/ryct.2020200110.

5. Zou L, Ruan F, Huang M, et al. SARS-CoV-2 viral load in upper respiratoryspecimens of infected patients. N Engl J Med. 2020;382:1177-9. doi:10.1056/NEJMc2001737.

6. Kimball A, Hatfield KM, Arons M, et al. Public Health - Seattle \&King County. Asymptomatic and presymptomatic SARS-CoV-2 infectionsin residents of a long-term care skilled nursing facility KingCounty, Washington, March 2020. MMWR Morb Mortal Wkly Rep. 2020;69:377-81. doi:10.15585/mmwr.mm6913e1.

7. Choudry FA, Hamshere SM, Rathod KS, et al. High Thrombus Burden in Patients With COVID-19 Presenting With ST-Segment Elevation Myocardial Infarction. J Am CollCardiol. 2020;76:1168-76. doi:10.1016/j.jacc.2020.07.022.

8. Modin D, Claggett B, Sindet-Pedersen C, et al. Acute COVID-19 and the Incidence of Ischemic Stroke and Acute Myocardial Infarction. Circulation. 2020;142:2080-2082. doi:

0.1161/CIRCULATIONAHA.120.050809. 
9. Alpert JS, Thygesen K, Antman E, Bassand JP. Myocardial infarction redefined-a consensus document of The Joint European Society of Cardiology/American College of Cardiology Committee for the redefinition of myocardial infarction. J Am CollCardiol. 2000;36:959-69. doi:10.1016/s07351097(00)00804-4.

10. Sianos G, Papafaklis MI, Serruys PW. Angiographic thrombus burden classification in patients with ST-segment elevation myocardial infarction treated with percutaneous coronary intervention. $J$ Invasive Cardiol. 2010;22(10 Suppl B):6B-14B.

11. Ibanez B, James S, Agewall S, et al.; ESC Scientific Document Group. 2017 ESC Guidelines for the management of acute myocardial infarction in patients presenting with ST-segment elevation: The Task Force for the management of acute myocardial infarction in patients presenting with STsegment elevation of the European Society of Cardiology (ESC). Eur Heart J. 2018 Jan 7;39(2):119177.

12. Neumann FJ, Sousa-Uva M, Ahlsson A, et al.; 2018 ESC/EACTS Guidelines on myocardial revascularization. Eur Heart J. 2019 Jan 7;40(2):87-165.

13. Ge J, Schäfer A, Ertl G, Nordbeck P. Thrombus Aspiration for ST-Segment-Elevation Myocardial Infarction in Modern Era: Still an Issue of Debate? Circ Cardiovasc Interv. 2017 Oct;10(10):e005739. doi: 10.1161/CIRCINTERVENTIONS.117.005739.

14. van't Hof AW, Liem A, Suryapranata H, Hoorntje JC, de Boer MJ, Zijlstra F. Angiographic assessment of myocardial reperfusion in patients treated with primary angioplasty for acute myocardial infarction: myocardial blush grade. Zwolle Myocardial Infarction Study Group. Circulation. 1998;97:2302-6.

15. Mitchell C, Rahko PS, Blauwet LA, Canaday B, Finstuen JA, Foster MC, Horton K, Ogunyankin KO, Palma RA, Velazquez EJ. Guidelines for Performing a Comprehensive Transthoracic Echocardiographic Examination in Adults: Recommendations from the American Society of Echocardiography. J Am Soc Echocardiogr. 2019;32:1-64.

16. Westblade LF, Brar G, Pinheiro LC, et al. SARS-CoV-2 Viral Load Predicts Mortality in Patients with and without Cancer Who Are Hospitalized with COVID-19. Cancer Cell. 2020;38:661-71.e2. doi:10.1016/j.ccell.2020.09.007.

17. Kampinga MA, Nijsten MWN, Gu YL, et al. Is the myocardial blush grade scored by the operator during primary percutaneous coronary intervention of prognostic value in patients with ST-elevation myocardial infarction in routine clinical practice? Circ Cardiovasc Interv. 2010;3:216-23.

18. Rehman AU, Malik J, Javed N, et al. Myocardial blush grade: a determinant of left ventricular ejection fraction and adverse outcomes in STEMI. Scott Med J. 2020 Jul 6. doi:10.1177/0036933020941260.

19. Kwong JC, Schwartz KL, Campitelli MA, et al. Acute Myocardial Infarction after Laboratory-Confirmed Influenza Infection. N Engl J Med. 2018;378:345-53.

20. Wang D, Hu B, Hu C, et al. Clinical Characteristics of 138 Hospitalized Patients with 2019 Novel Coronavirus-Infected Pneumonia in Wuhan, China. J Am Med Assoc. 2020;323:1061-9. 
21. Ruan Q, Yang K, Wang W, Jiang L, Song J. Clinical predictors of mortality due to COVID-19 based on an analysis of data of 150 patients from Wuhan, China. Intensive Care Med. 2020;46:846-8.

22. Yang $X, Y u Y, X u$ J, et al. Clinical course and outcomes of critically ill patients with SARS-CoV-2 pneumonia in Wuhan, China: a single-centered, retrospective, observational study. Lancet Respir Med. 2020;8:475-81.

23. Kamel MH, Yin W, Zavaro C, Francis JM, Chitalia VC. Hyperthrombotic Milieu in COVID-19 Patients. Cells. 2020;9:2392.

24. Petrovic V, Radenkovic D, Radenkovic G, Djordjevic V, Banach M. Pathophysiology of Cardiovascular Complications in COVID-19. Front Physiol. 2020;11:575600.

25. Beyrouti R, Adams ME, Benjamin L, et al. Characteristics of ischaemic stroke associated with COVID19. J Neurol Neurosurg Psychiatry. 2020;91:889-91. doi.org/10.1136/jnnp-2020-323586.

26. Paranjpe I, Fuster V, Lala A, et al. Association of Treatment Dose Anticoagulation with In-Hospital Survival Among Hospitalized Patients with COVID-19. J Am. 2020;76:122-4. http://dx.doi.org/10.1016/j.jacc.2020.05.001. CollCardiol .

27. Sardu C, Gambardella J, Morelli MB, Wang X, Marfella R, Santulli G. Hypertension, Thrombosis, Kidney Failure, and Diabetes: Is COVID-19 an Endothelial Disease? A Comprehensive Evaluation of Clinical and Basic Evidence. J Clin Med. 2020 May 11;9(5):1417. doi: 10.3390/jcm9051417.

28. Marfella R, Paolisso P, Sardu C, Bergamaschi L, D'Angelo EC, Barbieri M, Rizzo MR, Messina V, Maggi P, Coppola N, Pizzi C, Biffi M, Viale P, Galié N, Paolisso G. Negative impact of hyperglycaemia on tocilizumab therapy in Covid-19 patients. Diabetes Metab. 2020 Oct;46(5):403-5. doi:10.1016/j.diabet.2020.05.005.

29. Ibanez Borja, James S, Agewall S, et al. 2017 ESC Guidelines for themanagement of acutemyocardial infarction in patients presenting with ST-segment elevation. Eur Heart J. 2018;39:119-77.

30. Blumenstein J, Kriechbaum SD, Leick J, et al. Outcome of thrombus aspiration in STEMI patients: a propensity score-adjusted study. J ThrombThrombolysis. 2018 Feb;45(2):240-9.

31. Angerås $\mathrm{O}$, Haraldsson I, Redfors B, et al. Impact of Thrombus Aspiration on Mortality, Stent Thrombosis, and Stroke in Patients With ST-Segment-Elevation Myocardial Infarction: A Report From the Swedish Coronary Angiography and Angioplasty Registry. J Am Heart Assoc. 2018 Jan;9(1):e007680. doi:10.1161/JAHA.117.007680. 7 ) .

32. Blasco A, Coronado MJ, Hernández-Terciado F, et al. Assessment of Neutrophil Extracellular Traps in Coronary Thrombus of a Case Series of Patients With COVID-19 and Myocardial Infarction. JAMA Cardiol. 2020 Dec 29:e207308. doi: 10.1001/jamacardio.2020.7308.

\section{Figures}



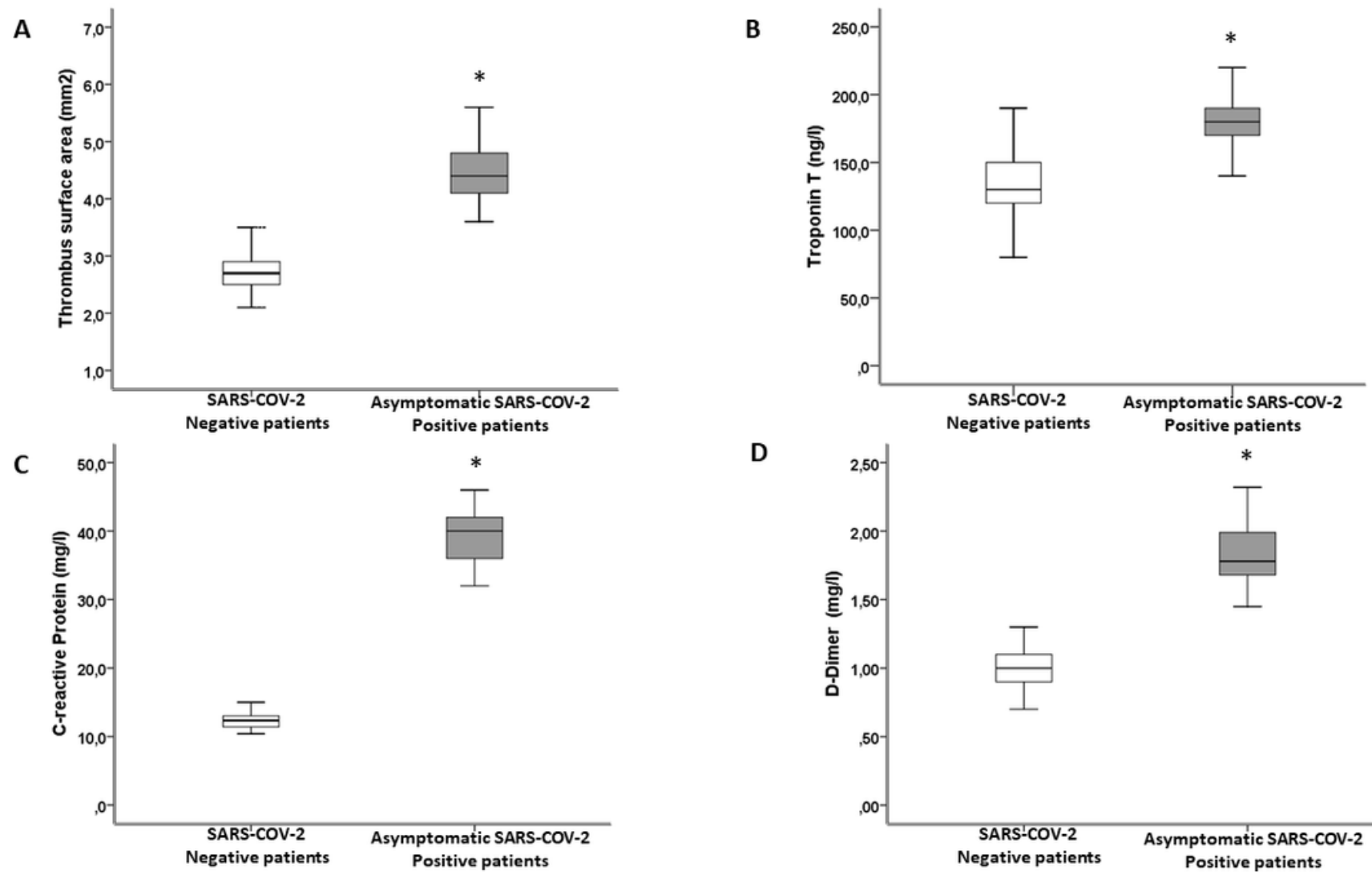

D

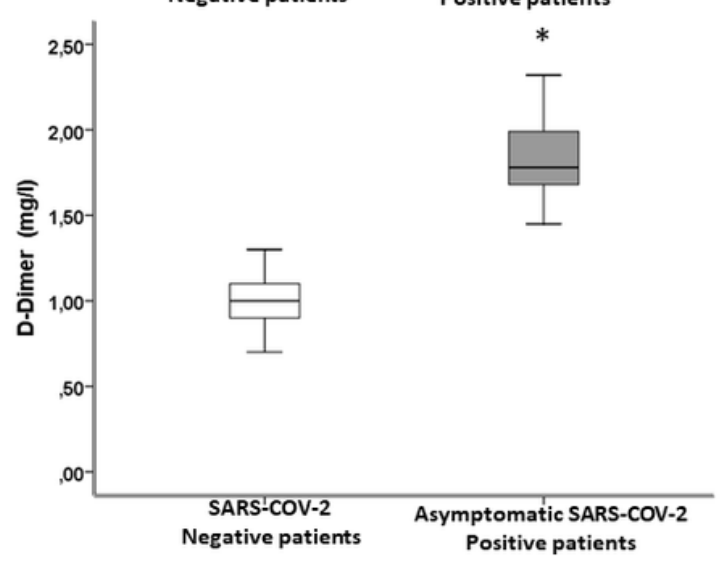

\section{Figure 1}

Panel A, thrombus surface areas (mm2) from 130 SARS-COV-2 negative STEMI patients and 46 asymptomatic SARS-COV-2 positive STEMI patients (Boxplot, a plot type that displays the median, 25th, and 75th percentiles and range). Panel $B$, troponin T levels from 130 SARS-COV-2 negative STEMI patients and 46 asymptomatic SARS-COV-2 positive STEMI patients. Panel C, C-reactive protein levels from 130 SARS-COV-2 negative STEMI patients and 46 asymptomatic SARS-COV-2 positive STEMI patients. Panel D, D-dimer levels from 130 SARS-COV-2 negative STEMI patients and 46 asymptomatic SARS-COV-2 positive STEMI patients. Data are mean \pm SD. ${ }^{*}<<0.01$ vs.asymptomatic SARS-COV-2 positive STEMI patients. 

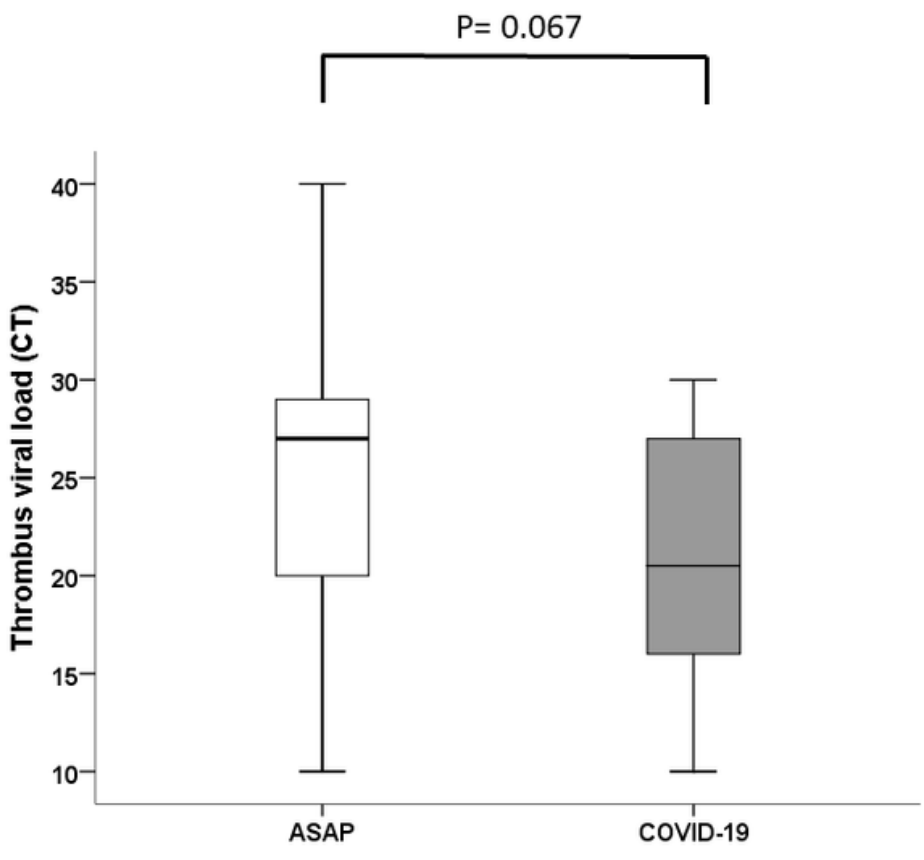

\section{Figure 2}

Cycle thresholds in thrombi from asymptomatic SARS patients and COVID-19 patients.

A

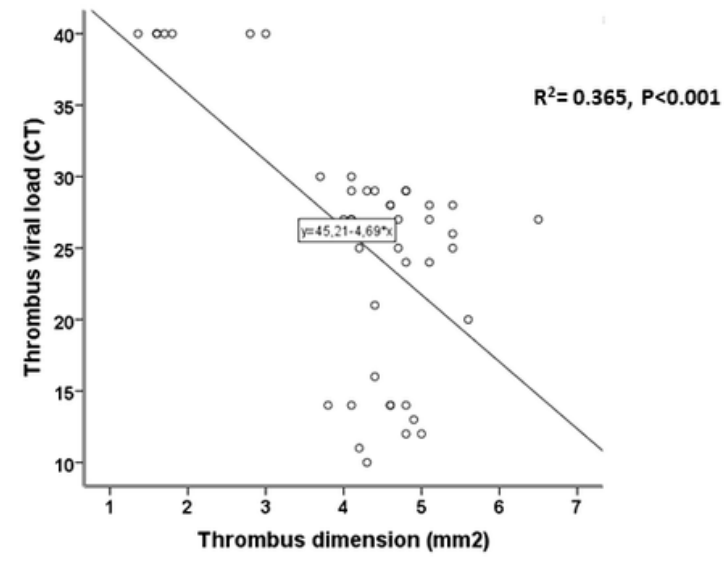

C

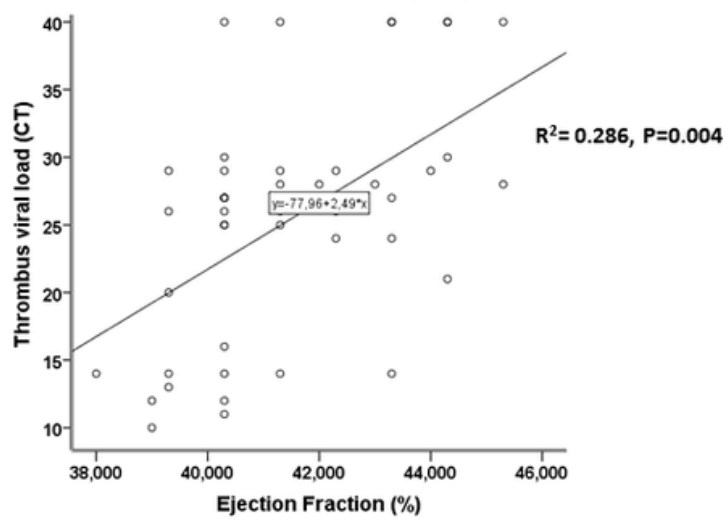

B

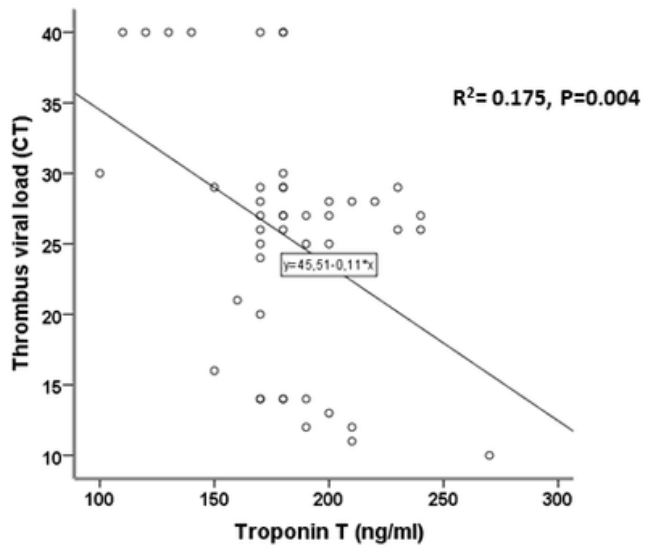

D

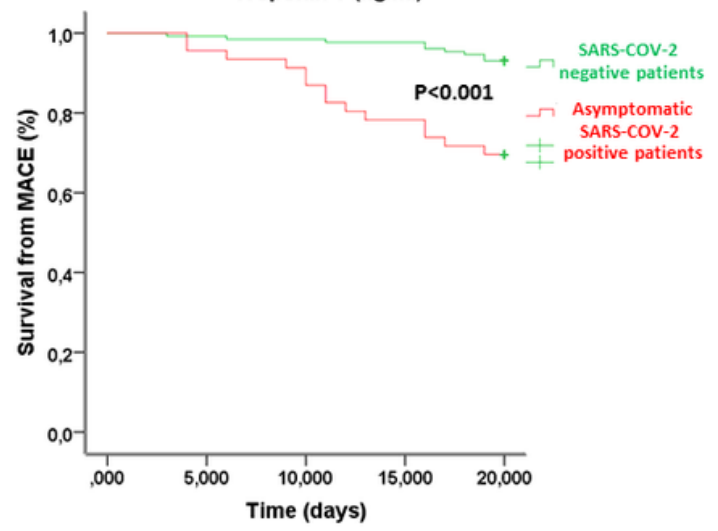




\section{Figure 3}

Panel A, regression analysis between thrombus SARS-COV-2 virus load and thrombus dimensions in asymptomatic SARS-COV-2 positive STEMI patients $(n=46)$. Panel $B$, regression analysis between thrombus SARS-COV-2 virus load and troponin T levels in asymptomatic SARS-COV-2 positive STEMI patients $(n=46)$.Panel $C$, regression analysis between thrombus SARS-COV-2 virus load and ejection fraction in asymptomatic SARS-COV-2 positive STEMI patients $(n=46)$. Panel $D$, Risk-adjusted KaplanMeyer analysis curves showing survival from MACE through follow-up in STEMI patients in asymptomatic SARS-COV-2 positive and SARS-COV-2 negative patients.

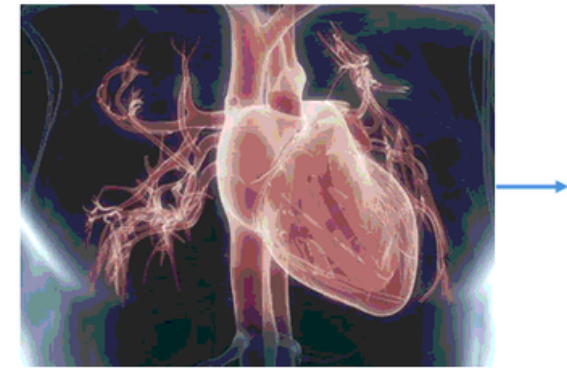

SARS-COV-2 negative patients

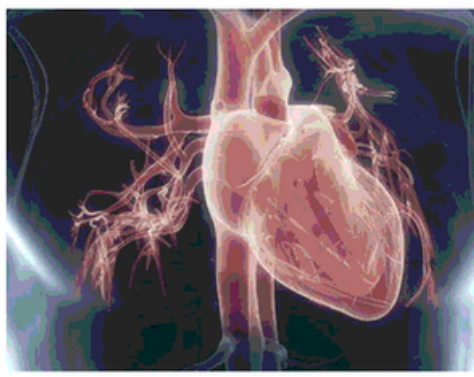

Asymptomatic SARS-COV-2 positive patients

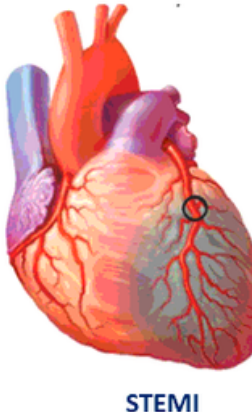

STEMI
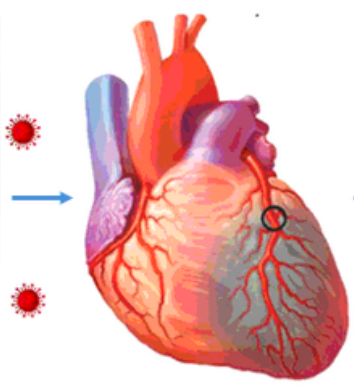

STEMI

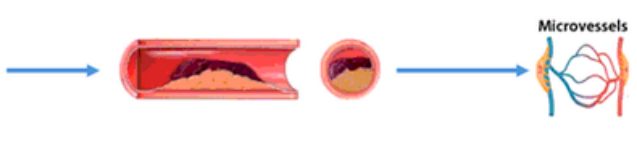

Coronary thrombus

Blush grade $>2$

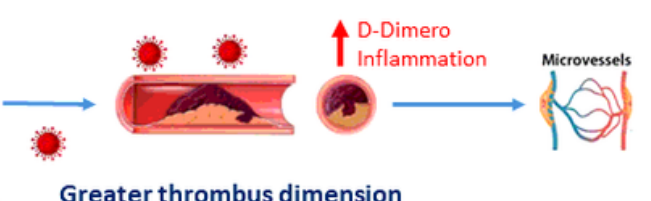

Coronary thrombus

Blush grade $<2$

\section{Figure 4}

The hypothesis of the thrombus SARS-COV-2 colonization effects on myocardial microvascular bed perfusion in asymptomatic SARS-COV-2 STEMI patients. We evaluated thrombus viral load and thrombus burden and their impact on microvascular bed perfusion in the infarct area (Blush grade, MBG) in asymptomatic SARS-COV-2 positive compared to SARS-COV-2 negative STEMI patients. In asymptomatic SARS-COV-2 positive patients presenting with STEMI, there is strong evidence towards higher thrombus viral load, dimension, and poorer MBG. 